
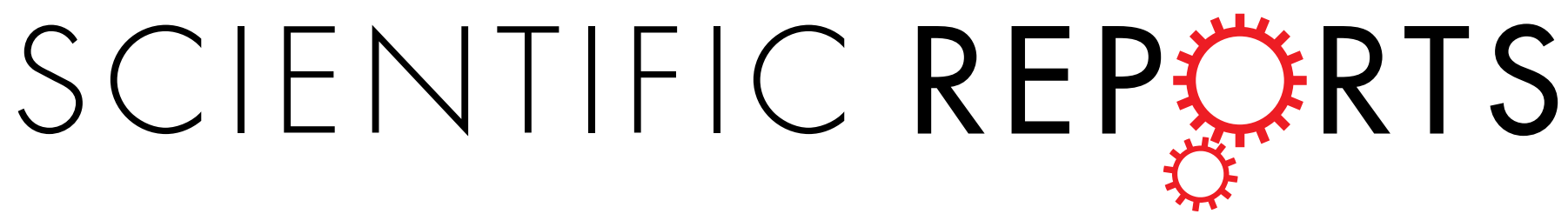

\title{
OPEN Correlations of charge neutrality level with electronic structure and p-d hybridization
}

Received: 28 July 2016

Accepted: 09 December 2016

Published: 19 January 2017

\section{Arkaprava Das ${ }^{1}$, Subodh K. Gautam¹, D. K. Shukla² \& Fouran Singh ${ }^{1}$}

The formation of charge neutrality level (CNL) in highly conducting Cadmium oxide (CdO) thin films is demonstarted by the observed variation in the band gap upon annealing and doping. It may be explained by the observation that Tin ( $\mathrm{Sn}$ ) doping breaks the perfect periodicity of $\mathrm{CdO}$ cubic crystal structure and creates virtual gap states (ViGS). The level of local CNL resides at the branch point of ViGS, making the energy at which native defect's character changes from predominantly donor-like below CNL to predominantly acceptor-like above the CNL and a schematic band diagram is developed to substantiate the same. Further investigations using soft $x$-ray absorption spectroscopy (SXAS) at Oxygen and Cadmium edges show the reduction of $\mathrm{Sn}^{4+}$ to $\mathrm{Sn}^{2+}$. The analysis of the spectral features has revealed an evidence of $p$ - $d$ interaction between $\mathrm{O} 2 p$ and $\mathrm{Cd} 4 d$ orbitals that pushes the valence band minima at higher energies which is symmetry forbidden at $r$ point and causing a positive valance band dispersion away from the zone centre in the $r \sim \mathrm{L}, \mathrm{K}$ direction. Thus, origin of the CNL is attributed to the high density of the Oxygen vacancies as confirmed by the change in the local electronic structure and $p$ - $d$ hybridization of orbitals.

Cadmium oxide $(\mathrm{CdO})$ is an $\mathrm{n}$ type degenerate semiconductor with direct band gap of $2.2 \mathrm{eV}^{1}$ and non-stoichiometric composition due to the presence of Cd interstitials ${ }^{2}$ or oxygen vacancies ${ }^{3}$ which act as doubly charge donors ${ }^{4}$. It crystallizes in rocksalt phase and shows transparency almost in the whole visible region of the electromagnetic spectrum ${ }^{1}$. It has an intrinsic high electrical conductivity with high optical transmittance. CdO is a well known wide band gap semiconductor for exhibiting very high intrinsic mobility without additional doping to the lattice. Due to its distinguishable features mentioned above in recent years CdO has attracted the attention of research community. CdO is potentially an ideal transparent conducting oxide (TCO) for optoelectronic devices operating at lower wavelengths ${ }^{5}$ region, flat panel display ${ }^{6}$ and thin film photovoltaics. It can be used for full range photovoltaic applications with a pseudo alloy developed with $\mathrm{ZnO}$. CdO is also useful for various versatile applications like transparent electrodes, gas sensors, liquid crystal display, photodiodes, and phototransistors. In order to enhance the electrical conductivity and band gap, $\mathrm{Sn}$ doping has been used as its ionic radii of $\mathrm{Sn}^{4+}$ is less than $\mathrm{Cd}^{2+}$ and electronegativity is little bit higher than $\mathrm{Cd}$. So replacement of $\mathrm{Cd}$ ions by $\mathrm{Sn}$ ions is expected after doping.

Raman spectroscopy is one of the most prominent tools for the investigation and characterization of semiconductor material. First order Raman modes are forbidden for rocksalt structures like CdO. In the 1960s, zone centre transverse-optical (TO) and longitudinal-optical (LO) phonon modes of $\mathrm{CdO}$ were suggested to be located at $262 \mathrm{~cm}^{-1}$ and $523 \mathrm{~cm}^{-1}$ respectively by infrared measurements ${ }^{7}$. We performed Raman scattering on annealed thin film prepared by solgel method and find 3 main peaks i.e. one TO mode centred at $290 \mathrm{~cm}^{-1}$ and two LO modes centred at $472 \mathrm{~cm}^{-1}$ and at $952 \mathrm{~cm}^{-1}$. The dependence of Raman spectra on annealing temperature and doping have been discussed in detail. It is also known that the study of the hybridization of orbitals in CdO exhibits interesting phenomenon for the understanding the fundamental investigations of Physics. It may also be noted that the information about Bohr exciton radius of $\mathrm{CdO}$ is not well reported in the literature due to the peculiar behaviour of the band structure, which has been ascribed to the electronic structure. Therefore, the present manuscript is focused on the effect of crystallite size and doping on the electronic structure and is investigated within the frame work of charge neutrality level (CNL).

${ }^{1}$ Inter University Accelerator Centre, Aruna Asaf Ali Marg, New Delhi-110067, India. ${ }^{2}$ UGC-DAE Consortium for Scientific Research, University Campus, Khandwa Road, Indore 452017, India. Correspondence and requests for materials should be addressed to F.S. (email: fouran@gmail.com) 


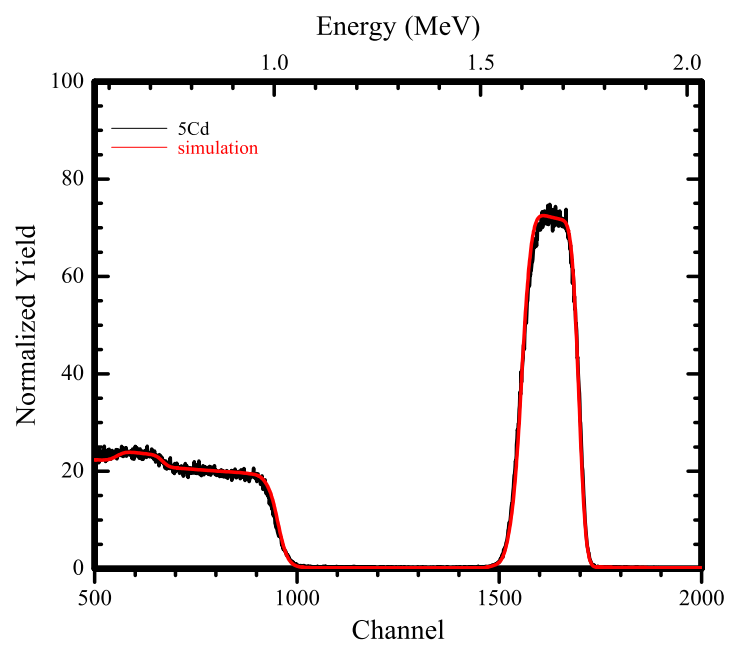

Figure 1. Rutherford backscattering spectrum for 5Cd thin film.

\section{Methods}

Undoped and doped $\mathrm{CdO}$ films were deposited on the corning glass, silica and silicon substrates using sol-gel spin coating technique. Cadmium acetate dihydrate $\left[\mathrm{Cd}\left(\mathrm{CH}_{3} \mathrm{COO}\right)_{2} \cdot 2 \mathrm{H}_{2} \mathrm{O}\right]$ and Tin chloride pentahydrate were taken as a source of $\mathrm{Cd}$ and $\mathrm{Sn}$ in the solution. 2- Methoxy ethanol and Di-ethanolamine (DEA) were used as a solvent and sol stabilizer. In case of undoped solution the precursor was prepared with desired molarity by mixing Cadmium acetate dehydrate with 2- methoxy ethanol and stirred for 10 hours with the help of a magnetic stirrer. In case of $1 \% \mathrm{Sn}$ doped $\mathrm{CdO}$ the precursor solution with intended molarity was prepared by mixing proper amount of Tin chloride pentahydrate with 2- methoxy ethanol. This precursor solution was stirred for 20 minutes. After that proper amount of Cadmium acetate dihydrate was added to the stirred solution which was stirred for 10 hours. Both the solutions were prepared at room temperature. For proper gelation, prepared solutions were aged for 2 days. As a precautionary measure the corning glass and silica substrate were rinsed in acetone and deionised water solution in an ultrasonic bath for 10 minutes. The silicon substrates were rinsed in $5 \%$ hydrofluoric acid solution (acid + deionised water) in an ultrasonic bath for 10 minutes and subsequently washed by deionised water to remove the oxidised layer. The films were deposited with the help of a spin coater with a speed of 2800 revolution per minute (rpm) for 30 seconds. The films were dried after each coating using a hot plate at $200^{\circ} \mathrm{C}$ for evaporating organic residuals. This process was repeated for 12 times to get a homogeneous distributed film with desired thickness. The prepared undoped films were annealed at 400 and $500{ }^{\circ} \mathrm{C}$ in the oxygen environment respectively. The Sn doped films were annealed at $400^{\circ} \mathrm{C}$ at oxygen environment. Annealing was done using a tubular furnace.

The structural characterization of $\mathrm{Sn}_{\mathrm{x}} \mathrm{Cd}_{1-\mathrm{x}} \mathrm{O}$ thin films was done by using Bruker High resolution X-Ray diffractometer system in the $2 \theta$ range $30^{\circ}-70^{\circ}$ with $\mathrm{Cu} \mathrm{K}_{\alpha}$ radiation with step size of $0.02^{\circ}$. The micro-Raman measurements were carried out using Renishaw InVia Raman microscope under the excitation by $514.5 \mathrm{~nm}$ argon ion laser operating at $50 \mathrm{~mW}$ of power. The thickness of films were determined using Rutherford Backscattering Spectrometry (RBS) using $2 \mathrm{MeV} \mathrm{He}^{+}$ions as incident beam at back scattering detection angle of $165^{\circ}$. Surface morphology and compositional analysis of the films were investigated by Scanning Electron Microscopy (SEM) with MIRA $\backslash$ TESCAN instrument using energy of $25 \mathrm{KeV}$. For recording transmittance and absorption spectra of the films, UV-Visible absorption measurements were carried out using Hitachi 3300 double beam spectrophotometer at room temperature. Surface topography measurements were carried out by atomic force microscopy (AFM) using NANAOSCOPE SPM II in tapping mode. The SXAS measurements of thin films at the O K and $\mathrm{Cd} \mathrm{M}_{4,5}$ edges were carried out in total electron yield (TEY) mode at SXAS beam line (BL-01) of the Indus- 2 synchrotron radiation source at Raja Ramanna Centre for Advanced Technology (RRCAT), India. For SXAS data processing Athena software package was used. These measurements were carried out in an ultra high vacuum $\sim 10^{-10}$ Torr. The carrier concentration and Hall mobility were measured by Van der Pauw Ecopia HMS-3000 Hall measurement system.

\section{Results and Discussions}

Structural Properties. Pure $\mathrm{CdO} 500^{\circ} \mathrm{C}$ annealed (5Cd) thin films were studied for their compositional analysis by RBS technique. The simulation was done by rump software. The thickness of the thin film was found to be $210 \mathrm{~nm}$ after simulation. The simulated spectrum of undoped $\mathrm{CdO}$ thin film had shown the proper stoichiometric ratio for the presence of $\mathrm{Cd}$ and $\mathrm{O}$ as their elemental atomic fraction was $\mathrm{Cd}-0.5$ and $\mathrm{O}-0.5$. However the interface between Si peak edge and lower energy edge of $\mathrm{Cd}$ is not very sharp. Fitting reveals a linear diffusion of $\mathrm{Cd}$ within the $\mathrm{Si}$ layer which is shown in Fig. 1 . There is no significance of this simulation for Sn doped thin films as the kinematic factor of $\mathrm{Sn}$ and $\mathrm{Cd}$ is very nearby to each other ${ }^{8}$. So RBS technique can't resolve those two elements.

Figure 2 shows the glancing angle X-ray diffraction (GAXRD) pattern for undoped $400^{\circ} \mathrm{C}(4 \mathrm{Cd}), 5 \mathrm{Cd}$ and $1 \%$ Tin doped $400^{\circ} \mathrm{C}(4 \mathrm{Cd}: \mathrm{Sn})$ annealed $\mathrm{CdO}$ thin films. The GAXRD pattern reveals that CdO thin films are polycrystalline in nature and shows a cubic structure (JCPDS: 78-0653) at room temperature. The pattern shows 


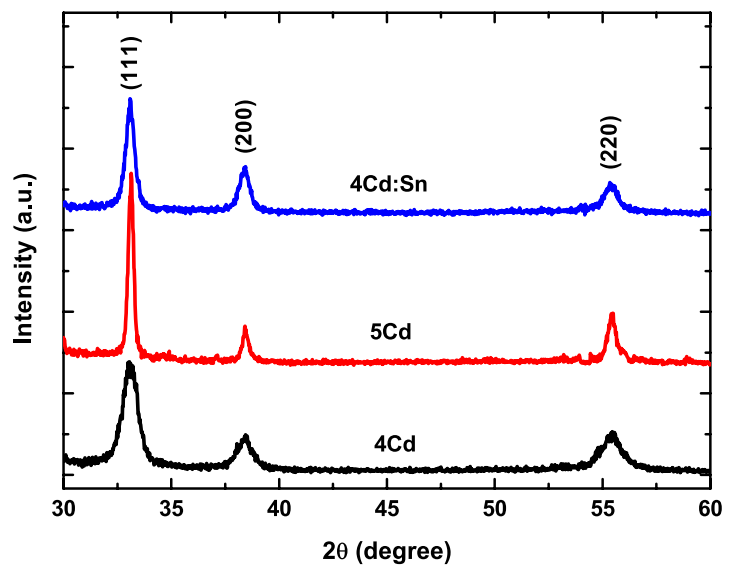

Figure 2. Grazing angle x-ray diffraction patterns for $4 \mathrm{Cd}, 5 \mathrm{Cd}, 4 \mathrm{Cd}$ :Sn thin films.

\begin{tabular}{|l|c|c|c|}
\hline Features & $\mathbf{4 0 0} \mathbf{C d O}$ & $\mathbf{5 0 0} \mathbf{C d O}$ & $\mathbf{1 S n}$ \\
\hline Particle diameter & $8 \mathrm{~nm}$ & $27 \mathrm{~nm}$ & $17 \mathrm{~nm}$ \\
\hline $2 \theta$ position (degree) & 33.14 & 33.13 & 33.22 \\
\hline mobility $\left(\mathrm{cm}^{2} / \mathrm{V}-\mathrm{s}\right)$ & 39.25 & 172.2 & 7.52 \\
\hline Band gap & $2.51 \mathrm{eV}$ & $2.36 \mathrm{eV}$ & $2.77 \mathrm{eV}$ \\
\hline Conductivity $\left(\mathrm{mho}^{\left.-\mathrm{cm}^{-1}\right)}\right.$ & $1.41 \mathrm{e} 02$ & $9.101 \mathrm{e} 02$ & $8.80 \mathrm{e} 01$ \\
\hline$r m s$ roughness & $4 \mathrm{~nm}$ & $15 \mathrm{~nm}$ & $3 \mathrm{~nm}$ \\
\hline Bulk carrier concentration $\left(\mathrm{cm}^{-3}\right)$ & $2.845 \mathrm{e} 19$ & $2.25 \mathrm{e} 19$ & $7.30 \mathrm{e} 19$ \\
\hline Resistivity $($ ohm-cm) & $5.78 \mathrm{e}-3$ & $7.06 \mathrm{e}-03$ & $1.13 \mathrm{e}-02$ \\
\hline Calculated BMS & $0.13 \mathrm{eV}$ & $0.11 \mathrm{eV}$ & $0.23 \mathrm{eV}$ \\
\hline
\end{tabular}

Table 1. Numerical values of different characterizations.

that diffraction peak intensity corresponding to (111) plane has increased for undoped 5Cd and 4Cd:Sn film compared to $4 \mathrm{Cd}$ thin film which is a signature of particle diameter increment. Applying Scherrer equations to the full width at half maxima (FWHM) of the (111) peaks?

$$
\mathrm{D}=\frac{\mathrm{k}_{*} \lambda}{\beta \cos \theta}
$$

Where $\boldsymbol{\lambda}$ is the $\mathrm{X}$-ray wavelength, $\theta$ is the Bragg's diffraction angle (half of the peak position angle), $\beta$ is the full width half maximum of the main peak in XRD pattern in radian. $\mathrm{k}$ is the shape factor generally its value is taken 0.9 . Using this equation average particle diameter is determined for different thin films. The values of $2 \theta$ position and average particle diameter are cited in Table 1 . There is almost no change in the $2 \theta$ value for the (111) peak for these thin films. The observed decrement in FWHM of (111) peak for 5Cd and 4Cd:Sn films compared to $4 \mathrm{Cd}$ film indicates the enhancement of average particle diameter. It is reported by B.J. Zheng et al. ${ }^{10}$ that with Tin doping particle diameters decrease. But in our case, Tin doping exhibits particle diameter enhancement. It is well known that $\mathrm{Cd}^{2+}, \mathrm{Sn}^{4+}, \mathrm{Sn}^{2+}$ ions have standard ionic radii of $0.97 \AA, 0.71 \AA$, and $1.12 \AA^{11}$, respectively. An increase in crystallite size is a signature of decrease of CdO stoichiometry by substitutional replacement of Cd with $\mathrm{Sn}$. Each $\mathrm{Cd}^{2+}$ ions are substituted by $\mathrm{Sn}^{2+}$ ions with reduction of $\mathrm{Sn}^{4+}$ via creating oxygen vacancies in the lattice. This replacement enhances the electron concentration which is further verified by Hall measurement and optical studies. Ionic radius of the $\mathrm{Sn}^{2+}$ is greater than $\mathrm{Cd}^{2+}$. So the replacement results a lattice expansion in CdO crystals and as a consequence crystallite growth has been observed. It is also noteworthy that the substitution of $\mathrm{Cd}^{2+}$ by $\mathrm{Sn}^{2+}$ not only changes the bond length but also changes the short range order parameter which might be another possible reason to produce a change in micro-structural strain. This change in micro-structural strain can be corroborated to the crystallization of polycrystalline thin films ${ }^{12}$. However, the $2 \theta$ position of (111) plane for $1 \mathrm{Sn}$ thin film in the XRD pattern remains unchanged. It is clear from the GAXRD pattern of Tin doped film that no additional diffraction peak for $\mathrm{SnO}_{2}$ has been formed. This proves that doping amount (1\%) in CdO lattice was within solubility limit. Moreover, $\mathrm{SnO}_{2}$ phase is reported to appear when doping limit is $7.6 \%$ or greater ${ }^{10}$.

Growth mechanism of CdO nanocrystallites. Generally nanocrystalline formation in thin films is dominated by nuclei formation, growth, and coalescence processes. At lower annealing temperature small crystallites get sufficient kinetic energy for initiation of coalescence process. If the nanoparticles diameter is limited within $5-10 \mathrm{~nm}$ range, cluster migration process takes place at lower growth temperature ${ }^{13}$. Ostwald ripening and sintering mechanism are dominated within $500^{\circ} \mathrm{C}-900^{\circ} \mathrm{C}$ range ${ }^{13}$. For $\mathrm{ZnO}$ crystallites, the growth kinetics is well 

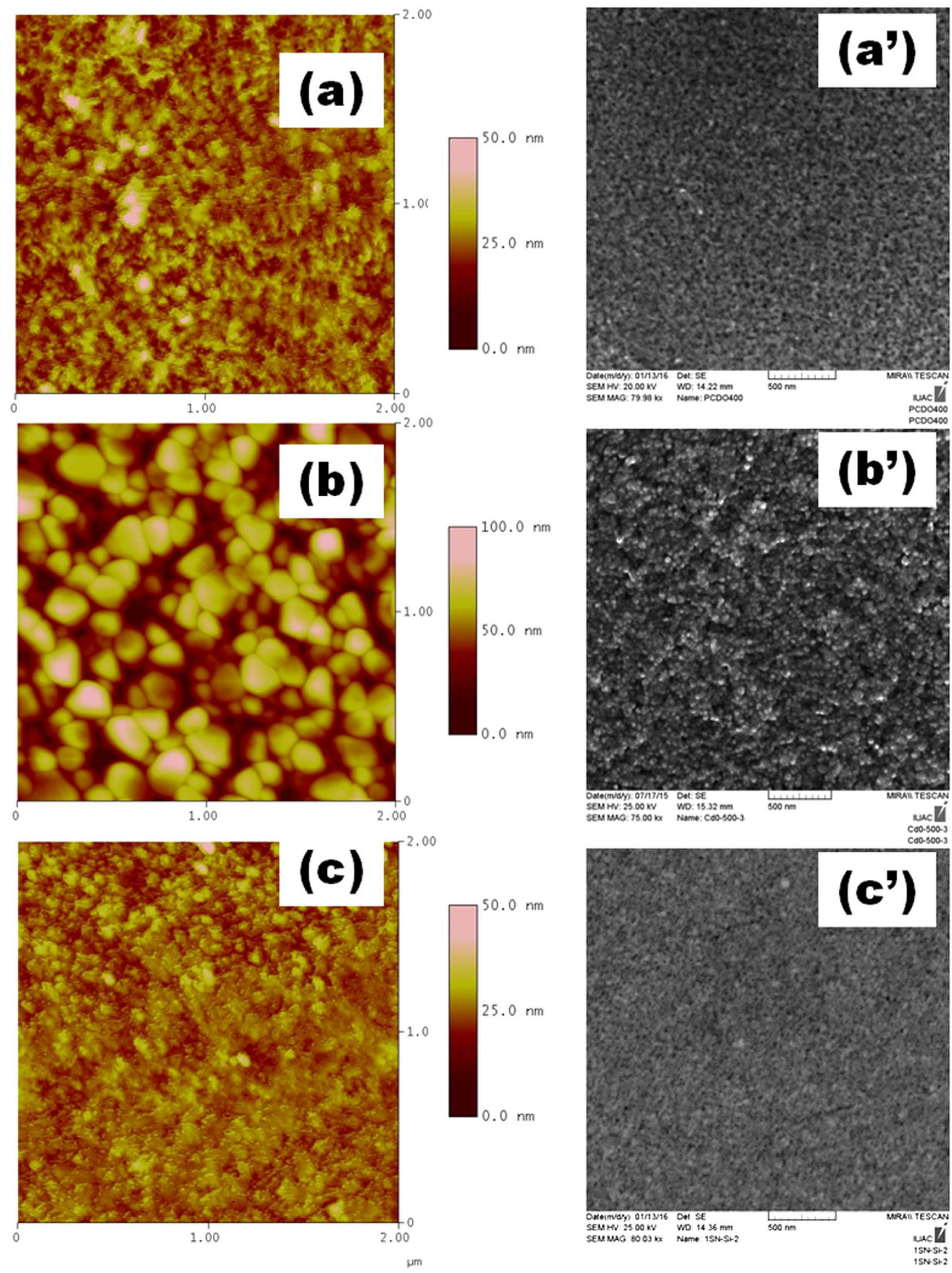

Figure 3. AFM and SEM images for 4Cd (a, a'), 5Cd (b, b’) and, $4 \mathrm{Cd}: \mathrm{Sn}\left(\mathbf{c}, \mathbf{c}^{\prime}\right)$ thin films.

explained by Wagner theory of Ostwald ripening ${ }^{14}$. Here coalescence process is expected to be dominated by cluster migration as the annealing temperature had not gone beyond $500^{\circ} \mathrm{C}$. It is well known that various kinds of defects are produced during the preparation of the thin films which induce stress in the films. During higher calcination temperatures the atoms in the lattice gain sufficient energy and get enough time to adjust their positions. As a consequence of that adjustment stress within the lattice tends to get relaxed.

From optoelectronic device application point of view, the surface properties of transparent conducting oxide influences the efficiency of the device and become a significant factor during device fabrication. In Fig. 3(a,b,c), (a,b'c') the AFM and SEM images of 4Cd, 5Cd and 4Cd:Sn thin films are presented. All the images show uniformity in thin film without any huge crack or dust particle. $4 \mathrm{Cd}: \mathrm{Sn}$ and $4 \mathrm{Cd}$ thin films are feebly crystallized and no voids are found. From the SEM images it is clear that with increasing annealing temperature, crystalline quality of the thin film enhances. This increment in average grain size with annealing temperature is further clarified by 

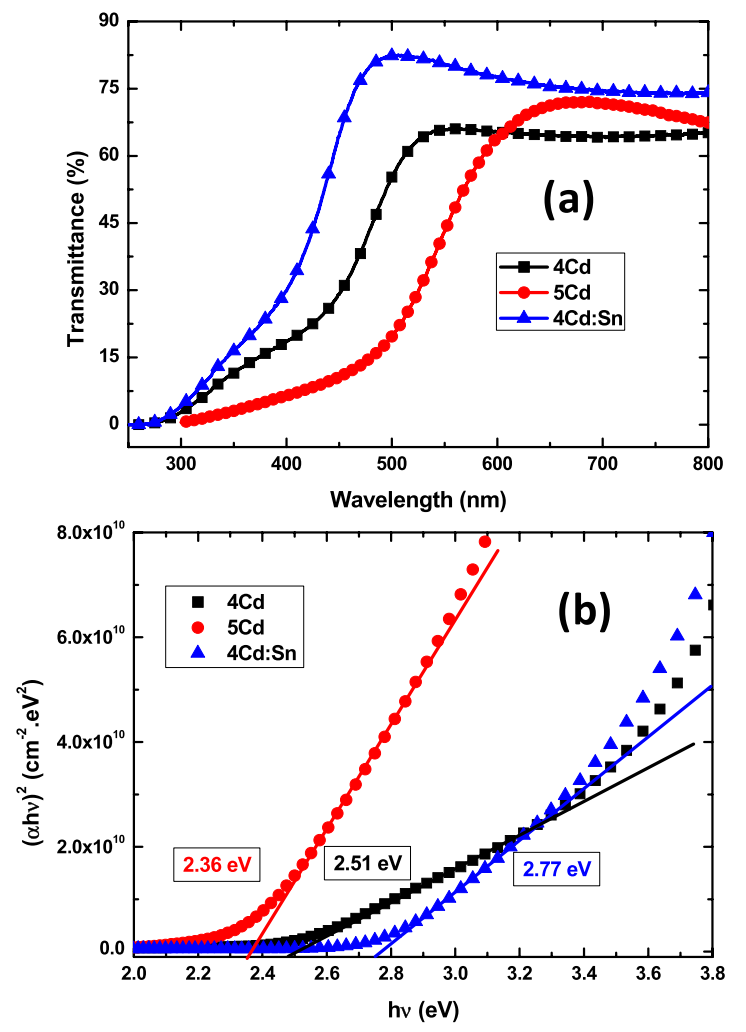

Figure 4. Transmittance spectra (a) and Tauc plot (b) for band gap calculation for 4Cd, 5Cd, and 4Cd:Sn thin films.

GAXRD pattern. This also results a decreases of the overall grain boundary area. The increment in root mean square (rms) roughness (calculated by nanoscope software) also verifies the decrease in grain boundary area which subsequently reduce the grain boundary scattering. The values of rms roughness and particle diameter are given in Table 1. The increment in rms roughness may lead to an enhancement of propagation loss for surface acoustic wave $(S A W)^{10}$ and degrade the efficiency of photovoltaic solar cells. Therefore, the increment in grain size and surface roughness may have opposite influences on the electrical and optical properties. There is not much change in roughness between $4 \mathrm{Cd}: S n$ and $4 \mathrm{Cd}$ thin films which means that there is not much change in the grain boundary area. Here, the roughness is in few $\mathrm{nm}$, so a little bit of change in the roughness would have very limited impact on the propagation of SAW.

Electrical properties. Hall measurement was carried out for the thin films in van der Pauw configuration at room temperature. The conductivity is reduced and carrier concentration increased drastically for Tin doped film. As mentioned earlier, each $\mathrm{Cd}^{2+}$ ion is substituted by a $\mathrm{Sn}^{2+}$ ion with reduction of $\mathrm{Sn}^{4+}$ via creating oxygen vacancies in the lattice which further enhances the carrier concentration and reduces the conductivity. It is quite clear that due to the substitution of $\mathrm{Cd}^{2+}$ by $\mathrm{Sn}^{4+}$, additional Oxygen vacancies are created at electrically active sites which liberate electrons and $\mathrm{Sn}^{4+}$ becomes $\mathrm{Sn}^{2+}$ in order to maintain the charge neutrality inside the lattice. The bulk carrier concentration of undoped $5 \mathrm{Cd}$ thin film is lower as-compared to the undoped $4 \mathrm{Cd}$ thin film. Due to higher annealing temperature in oxygen environment there might be a reduction in the concentration of Oxygen vacancies and Cd interstitials which is also observed from the sharper band edge in Tauc plot as shown in Fig. 4(b). This is a possible reason for reduction in carrier concentration in undoped 5Cd thin film. Enhancement in grain size improves the overall crystalline quality. An overall decrease in grain boundary fraction in the thin film reduces the grain boundary scattering which further reduces the resistivity. With Sn doping lattice distortion increases in $\mathrm{CdO}$ crystal subsequently an increase in grain boundary defects occurs. These defects produce scattering with the charge carriers which further reduces the mobility and conductivity of $1 \mathrm{Sn}$ thin film ${ }^{10}$. The values of resistivity and mobility are cited in Table 1.

From the above discussion it can be concluded that Tin would be an excellent choice as dopant in CdO for modifications of electrical properties as $\mathrm{Sn}$ can replace the $\mathrm{Cd}$ and enhances the carrier concentration which further reduces the conductivity but the doping concentration should be within the solubility limit which is about $4.4 \%$. This excellent property with Sn doping can be exploited in the formation of oxide based optoelectronic device $^{15}$.

Optical properties. The transmittance spectra are shown in Fig. 4(a). The transmittances for all the thin films are above $60 \%$ for wavelength more than $600 \mathrm{~nm}$ to $800 \mathrm{~nm}$. A prominent change in average transmittance has been observed in the $300 \mathrm{~nm}$ to $600 \mathrm{~nm}$ wavelength region. The transmittance in the absence of interference 
fringes is to be understood by Pankove analysis ${ }^{16}$. The transmittance in the visible region is found to vary from $55 \%$ to $75 \%$. The $5 \mathrm{Cd}$ thin film shows a lower percentage of transmission and sharper band edge in Tauc plot in Fig. 4(b) caused by the decrease in Oxygen vacancies with increasing annealing temperature. The red shift and the blue shift of the cut off wavelength in the transmittance spectra denotes a band gap shortening and band gap widening compared with the $4 \mathrm{Cd}$ thin film. The undoped thin film became reddish from yellowish nature with increasing annealing temperature. The energy band gaps of the thin films are calculated from the absorption spectra using Tauc plot shown in Fig. 4(b). If we want to correlate the band gap variation with the particle diameter via quantum confinement effect, the results are not compatible. The dependence of absorption coefficient $\alpha$ on the photon energy $(h \nu)$ is fitted using the relation ${ }^{17}$ :

$$
\alpha \mathrm{h} \nu=\mathrm{A}\left(\mathrm{h} \nu-\mathrm{E}_{\mathrm{g}}\right)^{\mathrm{n}}
$$

where $\mathrm{A}$ is a constant, $\mathrm{h} \nu$ is the photon energy, $\mathrm{E}_{\mathrm{g}}$ is the optical band gap of the thin film to be calculated. Here the exponent $\mathrm{n}$ depends on the type of allowed transition in the material. It has value equal to $1 / 2,2,3 / 2$ for allowed transitions, indirect transitions, and direct forbidden transitions respectively. CdO is direct energy band gap materials ${ }^{18}$. The extrapolation of the linear plot provides the value of band gap for direct allowed transition. The optical band gap values of thin films are found to be $2.51 \mathrm{eV}, 2.36 \mathrm{eV}, 2.77 \mathrm{eV}$ for $4 \mathrm{Cd}, 5 \mathrm{Cd}, 4 \mathrm{Cd}$ :Sn thin films, respectively. The errors in the band gap calculations are at third place of the decimal point $(+/-0.002 \mathrm{eV})$.

Carrier concentrations are different for these three samples and this can be attributed to the band gap variation through Burstein-Moss Shift (BMS) ${ }^{19,20}$. The observed higher band gap value for the Tin doped film comes from the filling of lower states of conduction band which is a common phenomenon for degenerate doped semiconductor. Due to BMS the enhanced value of the band gap is given by the following equation:

$$
\mathrm{Eg}=\mathrm{E}_{\mathrm{g}}^{0}+\Delta E_{\mathrm{g}}^{\mathrm{BM}}
$$

where $\mathrm{E}_{\mathrm{g}}{ }^{\circ}$ is the band gap of single crystal CdO and BMS is given by:

$$
\Delta \mathrm{E}_{\mathrm{g}}^{\mathrm{BM}}=\frac{h^{2}\left(3 \pi^{2} n_{e}\right)^{2 / 3}}{2 m_{v c}^{*}}
$$

and

$$
\frac{1}{m_{v c}^{*}}=\frac{1}{m_{v}^{*}}+\frac{1}{m_{c}^{*}}
$$

where $\mathrm{E}_{\mathrm{g}}{ }^{\circ}, m_{v}^{*}, m_{c}^{*}$ and $m_{v c}^{*}$ are the intrinsic band gap of an undoped semiconductor, the valence band effective mass, the conduction band effective mass and the reduced effective mass, respectively. The ratio of reduced effective mass to free electron mass $m_{v c}^{*} / m_{0}$ for pure CdO system is $0.274^{21}$ and the band gap of pure CdO system is $2.22 \mathrm{eV}$. It is noteworthy that the Bohr exciton radius for CdO is not reported in the literature so one can't draw any quantitative conclusion regarding the correlation of effective mass for $\mathrm{CdO}$ and its crystallite size. Using the equation (4) BMS for each thin film is calculated. The calculated values of BMS are cited in Table 1 but those values are not quantitatively compatible for all three thin films with the actual value of variation of band gap which come from the Tauc plot. Band gap enhancement for $5 \mathrm{Cd}$ compared to pure $\mathrm{CdO}$ band gap value is almost equal with the BMS i.e. $0.11 \mathrm{eV}$. However, the band gap of $4 \mathrm{Cd}$ and $4 \mathrm{Cd}: \mathrm{Sn}$ is enhanced by $0.29 \mathrm{eV}$ and $0.55 \mathrm{eV}$, respectively as compared to the bulk $\mathrm{CdO}$. Interestingly, for the $4 \mathrm{Cd}$ films a blue shift of $0.13 \mathrm{eV}$ is taken care by BMS and $0.16 \mathrm{eV}$ still remains. Similarly, for $4 \mathrm{Cd}: \mathrm{Sn}$ the band gap has been enhanced by $0.55 \mathrm{eV}$, within that only $0.23 \mathrm{eV}$ shift is taken care by BMS and $0.32 \mathrm{eV}$ still remains, which may not be explained by particle size effect as it is increased considerably as compared to $4 \mathrm{Cd}$ thin film.

Further, the band gap widening can be explained in the framework of charge neutrality level (CNL). CNL can be identified as the demarcation between the surface states that are predominantly donor-like (below) and acceptor-like (above) ${ }^{22}$. Due to Sn doping perfect periodicity of CdO cubic crystal structure has been broken as $\mathrm{Sn}^{4+}$ ions would pretend to behave as native donor type defect which is attributed to the generation of virtual gap states (ViGS). The level of local CNL resides at the branch point of ViGS, making the energy at which native defect's character changes from predominantly donor-like (valence band character) below CNL to predominantly acceptor-like (conduction band character) above the CNL ${ }^{23}$. So donor like ViGS are the surface states which reside below the local CNL. Due to prominent electronegativity and size mismatch between $\mathrm{O}$ and $\mathrm{Cd}$ atoms in CdO lattice the conduction band minima (CBM) at the $r$ point is significantly lower compared to the rest of the Brillouin zone ${ }^{24}$. Further, $p$ - $d$ interaction between $\mathrm{O} 2 p$ and $\mathrm{Cd} 4 d$ orbitals pushes the valence band minima $(\mathrm{VBM})$ at higher energies which is symmetry forbidden at $\mathrm{r}$ causing a positive valence band dispersion away from the zone centre in the $\mathrm{r} \sim \mathrm{L}, \mathrm{K}$ direction ${ }^{25}$. This explains well the reason for local CNL to reside intrinsically above CBM in CdO. So the donorlike ViGS below CNL from where each $\mathrm{Sn}^{2+}$ ion becomes $\mathrm{Sn}^{4+}$ by producing oxygen vacancies and the electrons from oxygen vacancies reach directly to the ViGS of conduction band as CNL lies above CBM leading to a large accumulation of electrons at the surface. Surface electron accumulation phenomenon in $\mathrm{CdO}$ has been well reported by King et al. ${ }^{26}$ Within the framework of amphoteric defect model the formation of donor type defects are more favourable when Fermi level is below compared to an energy level known as Fermi level stabilization energy $\left(\mathrm{E}_{\mathrm{FS}}\right)^{27}$. It is argued that there is an energy associated with each semiconductor which plays a role analogous to Fermi energy in metal ${ }^{28}$ and that energy is called CNL $\mathrm{E}_{\mathrm{B}}$ for complex band structure. Intrinsic tendency of Fermi level for any semiconductor system is to get coincident with $\mathrm{E}_{\mathrm{FS}}$ and $\mathrm{E}_{\mathrm{FS}}$ tends to follow $\mathrm{CNL}$ as the system goes into the saturation. Correlation of $\mathrm{E}_{\mathrm{FS}}$ and $\mathrm{CNL}$ is experimentally 


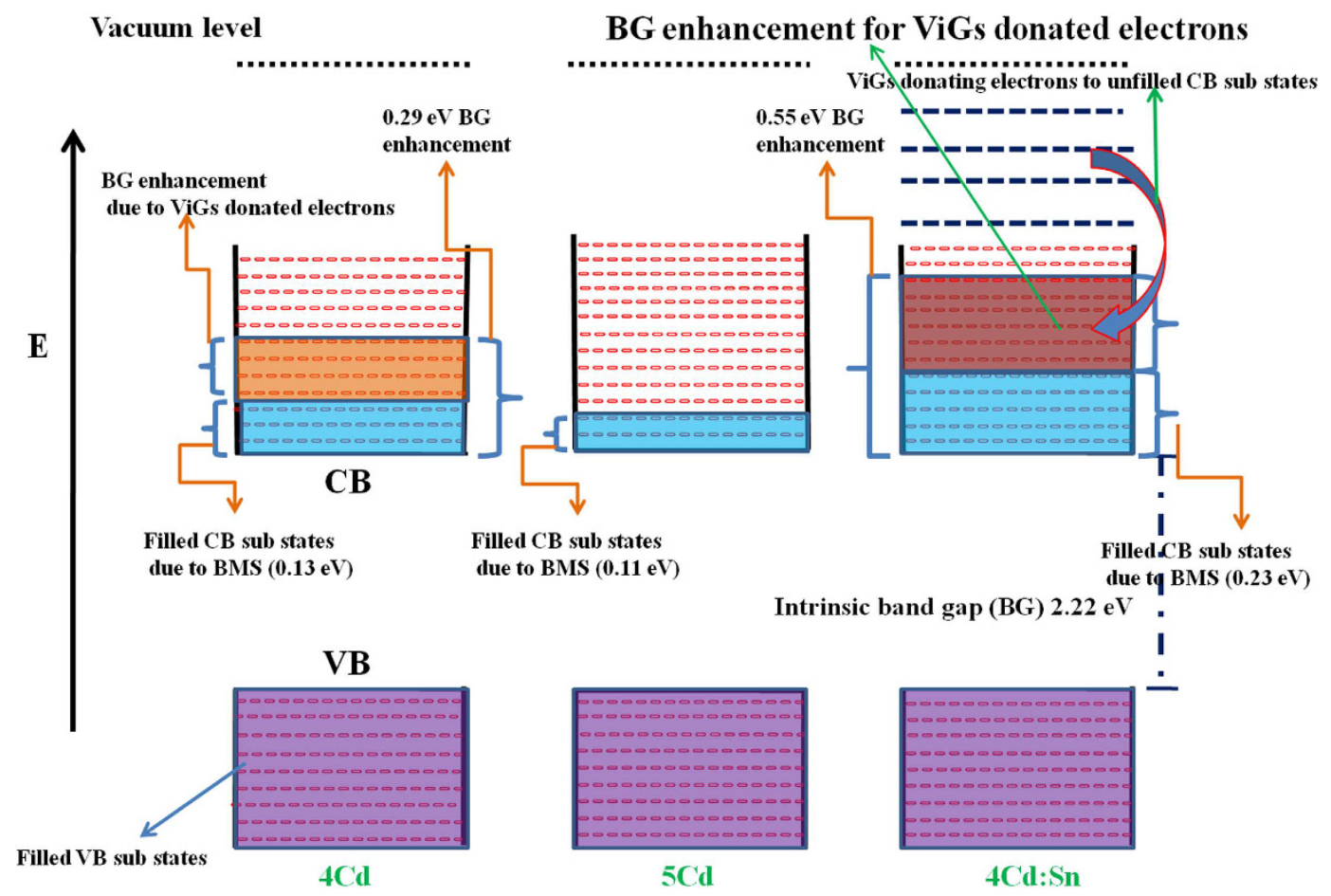

Figure 5. Developed schematic energy band diagram.

reported for GaAs by W. Walukiewicz ${ }^{29}$ and it correlates well with each other. It has been reported that CNL synonymously called midgap energy. It has been postulated as a reference point for pinning the Fermi energy at metal induced gap states which verifies our early discussion. From this postulate we can enunciate that in our case for $1 \mathrm{Sn}$ thin film virtual gap states have been generated due to incorporation of $\mathrm{Sn}$ and Fermi level pins taking CNL as reference energy level.

In covalent or semi-ionic semiconductor like $\mathrm{CdO}$ the coupling due to hybridization opens the optical band gap separating bonding from anti-bonding states. Incorporation of defects into the crystal drags bonding and anti-bonding states towards $\mathrm{CNL}$ which subsequently increase the density of states around $\mathrm{CNL}^{30}$. If $\mathrm{E}_{\mathrm{FS}}$ coincides with CNL, the formation energy of donor type defects and acceptor type defects become equal and further change in the carrier density will not be possible by creating native defects. This means that the system goes to the saturation condition as further creation of donor or acceptor type defects is not possible by creating additional defects or doping into the lattice. Thus, $\mathrm{E}_{\mathrm{FS}}$ favours the pinning of surface Fermi level above the CNL and resulting negative surface charge from occupied acceptor surface states which were unoccupied earlier when surface Fermi level was below than CNL. Due to overall alignment between surface and bulk region the Fermi level shifts little above CNL. As a consequence, for maintaining local charge neutrality there is an upward bending of the bands relative to the Fermi level and electron starts to deplete at the surface subsequently these surface depleted electrons fills the ViGS above CBM which explains the effective further band gap enhancement. This behaviour is well reported for $\mathrm{Sn}$ doped $\operatorname{In}_{2} \mathrm{O}_{3}$ by King et al. ${ }^{31}$. Band gap enhancement is shown schematically in the energy band diagram in Fig. 5. It is very difficult to depict the actual position of surface Fermi level, so in the energy band diagram the position of surface Fermi level has not been shown.

Raman spectroscopy. The space group symmetry of CdO rocksalt structure is $\mathrm{Fm} \overline{3} \mathrm{~m}$. It is well established that A1, E1 both are Raman and IR active branches, there symmetries are polar, doubly degenerated and split into TO and LO components with different frequencies. $\mathrm{E}_{2}(\mathrm{H})$ and $\mathrm{E}_{2}(\mathrm{~L})$ branches are non polar so they are Raman as well as IR inactive. Raman experiment was done in backscattering geometry, without any polarization analysis. The main features in Raman spectra have been shown Fig. 6. For 4Cd thin film a sharp peak is appearing centred at $290 \mathrm{~cm}^{-1}$, and two weaker bands are located at $793 \mathrm{~cm}^{-1}, 952 \mathrm{~cm}^{-1}$ respectively. A broad spanning structure from $300 \mathrm{~cm}^{-1}$ to $500 \mathrm{~cm}^{-1}$ is observed. Within that range a sharper intense peak centred at $472 \mathrm{~cm}^{-1}$ is observed. Theoretically it has been reported that CdO molecules have LO and TO phonon modes at $478(25) \mathrm{cm}^{-1}$ and at $262(3) \mathrm{cm}^{-1}$, respectively ${ }^{32,33}$. It is also well reported that peak centred at $952 \mathrm{~cm}^{-1}$ is LO phonon mode. Generally, in $\mathrm{CdO}$ rocksalt structure only second order Raman scattering is expected. The excitation wavelength for the Ar laser was $514.5 \mathrm{~nm}$ which is close to the band gap of CdO so the probability of first order Raman scattering can't be ruled out completely. It is well reported that the resonance effects are not important in determining the Raman spectrum line shape. According to the Raman selection rules for CdO rocksalt structure both LO and TO modes are dipole forbidden. All features over here in the Raman spectra can be attributed to the second-order scattering process in $\mathrm{CdO}^{34}$. The polarization vectors of incident and scattered light are parallel here, henceforth only $\mathrm{E}_{2}$ and $\mathrm{A}_{1}$ (LO and TO) modes would be allowed according to the Raman selection rule. The vibration of Cd and O 


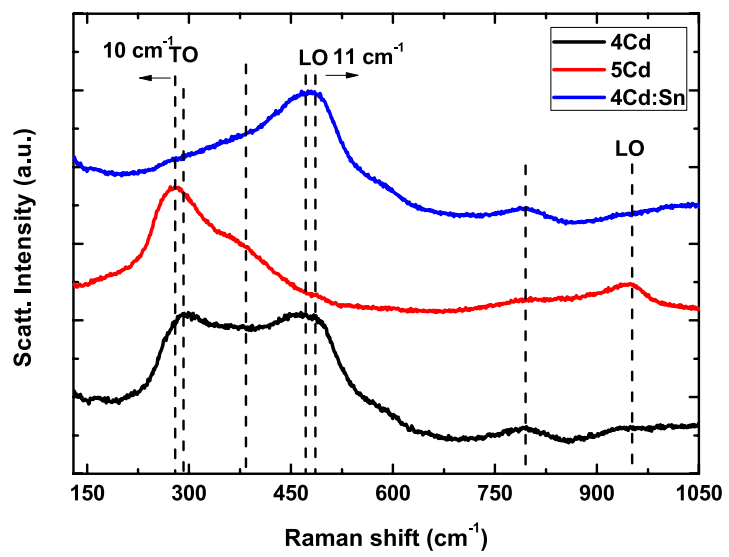

Figure 6. Raman spectra for $4 \mathrm{Cd}, 5 \mathrm{Cd}$, and $4 \mathrm{Cd}: \mathrm{Sn}$ thin films.

sub-lattices are related to the $\mathrm{E}_{2}$ (high) and $\mathrm{E}_{2}$ (low) modes which are nonpolar and sensitive to strain but not influenced by the carriers or electric field of the crystal ${ }^{35}$.

For 5Cd thin film, we observe that $290 \mathrm{~cm}^{-1}$ TO mode and $952 \mathrm{~cm}^{-1} \mathrm{LO}$ mode is getting sharper, $290 \mathrm{~cm}^{-1} \mathrm{TO}$ mode shows a red shift about $10 \mathrm{~cm}^{-1}$, a weak sub peak around $370 \mathrm{~cm}^{-1}$ has been evolved, the subpeak centred at $793 \mathrm{~cm}^{-1}$ becomes more intense without any shift in peak position and the LO Raman peak centred at $472 \mathrm{~cm}^{-1}$ smears out completely. For 4Cd:Sn thin film, LO phonon mode centred at $472 \mathrm{~cm}^{-1}$ becomes sharper and shows a blue shift of $11 \mathrm{~cm}^{-1}$ but the TO phonon mode centred at $290 \mathrm{~cm}^{-1}$ smears out completely. The weaker sub-peak centred at $793 \mathrm{~cm}^{-1}$ sustains well in this case. It is reported that $290 \mathrm{~cm}^{-1}$ TO phonon mode is a defect incorporated Raman peak for $\mathrm{CdO}$ rocksalt phase ${ }^{36}$. It is redundant to say that lot of impurities are present during thin film preparation which produces stress in the lattice and with annealing at higher temperature the atoms in the lattice get sufficient time and energy to readjust their positions. As a consequence stress tends to get relaxed. For $5 \mathrm{Cd}$ thin film impurities and defects decrease compared to $4 \mathrm{Cd}$ thin film. This is favourable for CdO rocksalt phase to get more stable and the $290 \mathrm{~cm}^{-1} \mathrm{TO}$ phonon mode for getting more prominent and intense in $5 \mathrm{Cd}$ thin film. $10 \mathrm{~cm}^{-1}$ red shift is a consequence of the relaxed lattice strain which can be called as softening of Raman phonon modes. Reducing band gap with increasing annealing temperature giving rise to a decrement in absorption coefficients for both excited and scattered radiation. This might be another possible reason for the sharper Raman peak at centred at $280 \mathrm{~cm}^{-1}$ for $5 \mathrm{Cd}$ thin film.

During Raman measurement the incident light was perpendicular to the surface of the thin films; hence the presence of LO phonon modes is quite obvious. For 4Cd:Sn thin film it is already enunciated that $\mathrm{Sn}^{2+}$ ions replaces the $\mathrm{Cd}^{2+}$ ions in the lattice which might act as the impurity centre and breaks the translational symmetry of the CdO lattice. As a consequence, $\mathrm{q}=0$ Raman selection rule is relaxed and phonons with $q \neq 0$ ( $\mathrm{q}$ is the wave vector) contribute to the Raman scattering away from the zone centre. In other words this relaxation in wave vector results in scattering by phonons in the host matrix which have wave vector far from the zone centre $^{37}$. Subsequently this leads to an asymmetric broadening and frequency shifts in phonon modes ${ }^{38}$. LO phonon modes of $\mathrm{CdO}$ results an atomic displacement along c-axis. The frequency shifts are proportionally related to the short range order parameter along c-axis, which is the distance between $\mathrm{Cd}$ and $\mathrm{O}$ atoms for pure $\mathrm{CdO}$ lattice. After replacement of $\mathrm{Cd}^{2+}$ ion by $\mathrm{Sn}^{2+}$ ion change in bond length as well as the short range order parameter takes place which deform the lattice and modulate the wave functions through deformation-potential scattering process $^{36}$. Therefore a complex interaction mechanism takes place here which may speculate the observed $11 \mathrm{~cm}^{-1}$ blue shift of LO phonon mode centred at $472 \mathrm{~cm}^{-1}$. This can be enunciated as the stiffening of that phonon mode.

Soft X-ray absorption spectroscopy (SXAS) studies. Soft X ray absorption spectra are recorded in TEY mode which has been measured from sample drain current. Figure 7 represents the experimental SXAS data for the Oxygen $\mathrm{K}$ edge and $\mathrm{Cd} \mathrm{M}_{4,5}$ edge ( normalized $\mu(\mathrm{E})$ versus photon energy $(\mathrm{eV})$ of $4 \mathrm{Cd}, 5 \mathrm{Cd}, 4 \mathrm{Cd}: \mathrm{Sn}$ thin films. Using ATHENA XAS data processing software (FEFF 6.0 code) the data were normalized and fitted to get the peak intensity ratio for Oxygen $\mathrm{K}$ edge which are cited in Table 2.

During Oxygen K edge peak fitting three different functions are taken i.e. arctangent, Gaussian, pseudo-voigt. From the fitting of $4 \mathrm{Cd}: \mathrm{Sn}$ film it is observed that the centre of the arctangent function shifts by $840 \mathrm{meV}$ below with respect to $4 \mathrm{Cd}$ thin film. This is direct indication that the CBM seems to be more towards Fermi level for $4 \mathrm{Cd}: \mathrm{Sn}$ films and thus in agreement with our previous discussion that Fermi level shifting in case of 4Cd:Sn thin film above $\mathrm{E}_{\mathrm{FS}}$. A higher electron concentration for $4 \mathrm{Cd}$ :Sn thin film also implies that conduction band is highly degenerate with a change in their effective mass. Henceforth the Fermi level is expected to be above conduction band and also to be pinned above CNL to maintain local charge neutrality. Moreover, the change in peak intensity ratio for $4 \mathrm{Cd}: \mathrm{Sn}$ as compared to $4 \mathrm{Cd}$ thin film can be attributed to the pinning of Fermi level above CNL for surface electron accumulation phenomena. After replacement of $\mathrm{Cd}^{2+}$ ion by $\mathrm{Sn}^{2+}$ ion there is a change in the bond length due to different electronegativity and ionic radii as well as in the crystal field which might be also a possible reason for the change in peak intensity ratio. There is not much change in the overall spectral profile for $4 \mathrm{Cd}$ and $4 \mathrm{Cd}: \mathrm{Sn}$ thin films. From this observation we can conclude that oxygen coordination in the neighbourhood of Cd 

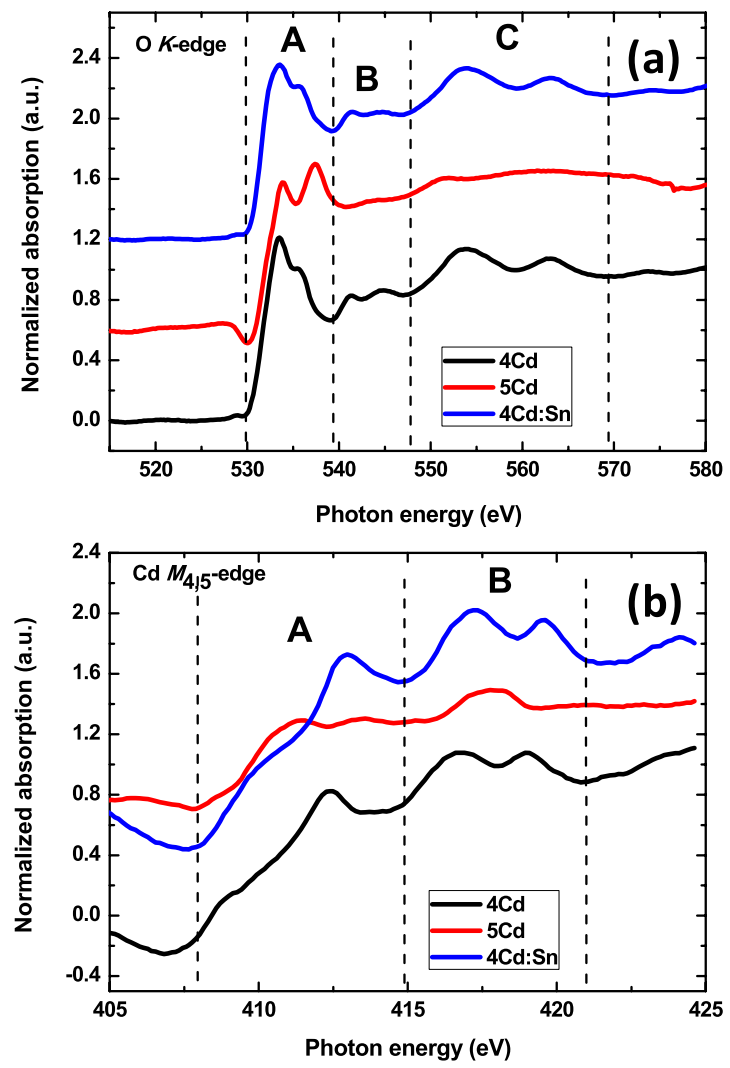

Figure 7. Soft X-ray absorption spectra for O k edge (a) and $\mathrm{Cd} \mathrm{M}_{4,5}$ edge (b) for 4Cd, 5Cd, 4Cd:Sn thin films.

\begin{tabular}{|l|c|c|c|}
\hline Sample & First peak & Second peak & Intensity ratio $\left(\mathbf{2}^{\text {nd }} / \mathbf{1}^{\text {st }}\right)$ \\
\hline 4Cd (O k edge $)$ & 1.197 & 0.747 & 0.62 \\
\hline 4Cd:Sn(O k edge $)$ & 1.066 & 0.963 & 0.90 \\
\hline 5Cd (O k edge $)$ & 0.555 & 1.086 & 1.96 \\
\hline
\end{tabular}

Table 2. Peak intensity ratios from SXAS analysis.

or $\mathrm{Sn}$ atoms are more or less same which is also a signature of almost equal amount native defects namely Oxygen vacancies for both the cases.

For the case of $5 \mathrm{Cd}$, a significant change in the peak intensity ratio as well as in the overall spectral profile for both Oxygen $\mathrm{K}$ and Cadmium $\mathrm{M}_{4,5}$ edges has been observed. It is well reported by I.N. Demchenko et al. that vacancies created into the Oxygen atom position has a deep impact on the overall spectral profile and edge shape ${ }^{39}$. Mainly spectral profiles are influenced by the native defects of first and second coordination shell. The change in spectral profile with increasing Oxygen vacancies has been theoretically well calculated by that group. Due to annealing at $500^{\circ} \mathrm{C}$ in Oxygen environment $\mathrm{CdO}$ lattice gets sufficient time to readjust their atomic positions which releases stress and the number of Oxygen vacancies also reduces as Oxygen diffuses inside the system. Therefore the spectrum of 5Cd looks like the profile where Oxygen vacancies are very less and due to limited resolution, $\mathrm{S}$ and $\mathrm{E}$ characters cannot be observed ${ }^{39}$. Hence, the changes in the intensity ratio of two first resonance and overall spectral profile can be attributed to the reduced number of Oxygen vacancies for 5Cd thin film. The same signature is obtained also from Cadmium $\mathrm{M}_{4,5}$ spectral profile.

The dipole transitions from Oxygen $1 \mathrm{~s}$ core state to unoccupied state with $\mathrm{p}$ character above Fermi level are allowed. In case of a purely ionic $\mathrm{Cd}^{2+} \mathrm{O}^{2-}$ bonding, the $2 p$ of Oxygen state must be occupied and the transition from $1 s$ to $2 p$ should not be observed. However, those transitions are experimentally visible which is a clear signature that $\mathrm{CdO}$ thin film is not purely ionic but of a mixed character ${ }^{24,40} \cdot \mathrm{Cd}_{\mathrm{M} 4,5}$ edges also gives the same signature that $\mathrm{CdO}$ thin films are of mixed character. The d electrons of $\mathrm{Cd}$ have effect not only on the band gap but also on the ground state properties such as equilibrium lattice parameter and cohesive energies ${ }^{41-43}$. The interaction between $\mathrm{Cd} 4 d$ and $\mathrm{O} 2 p$ determines the type of band gap of $\mathrm{CdO}$.

Particularly in CdO VBM is not at the centre of the Brillouin zone which leads to an indirect band gap contribution. This indirect band gap can be speculated to be the direct consequence of the hybridization of Cd $4 d$ with $\mathrm{O} 2 p$ states combined with octahedral point symmetry ${ }^{25}$. The hybridization of $\mathrm{O} 2 p$ states with Cd $4 d$ shallow electrons is well reported by Piper et al. ${ }^{24}$ by Xray emission spectroscopy (XES) which also supported by Xray photoelectron spectroscopy (XPS $)^{39}$. The signature of O $2 p$ hybridization with $\mathrm{Cd} 4 d$ states is not as sharp 
as reported by the theoretical calculations elsewhere for the XAS spectra ${ }^{39}$. We can speculate that the practical reason behind this difference between theoretical and experimental spectra would be the polycrystalline nature of thin films. Same signature of mixing can also be obtained from $\mathrm{Cd}_{\mathrm{M} 4,5}$ edges. For Oxygen $\mathrm{k}$ edges the peaks of the $\mathrm{B}$ and $\mathrm{C}$ region can be attributed to the interference effect of the multiple scattering signals. It is well reported from theoretical calculation by Demchenko et al. ${ }^{39}$ that with increasing cluster size there would be no generation of any new feature in the spectral profile apart from a little bit variations in the previously existing peaks for lower coordination.

\section{Conclusion}

In summary, the formations the virtual gap states as charge neutrality level are reported and its correlations with electronic structure have been discussed. This level is the energy at which native defect's character changes from donor-like to acceptor-like and it is well demonstrated by developing a schematic band diagram. Electronic structure using the soft $\mathrm{x}$-rays absorption spectroscopy at the Oxygen and Cadmium edge has been investigated and it has revealed the reduction of $\mathrm{Sn}^{4+}$ to $\mathrm{Sn}^{2+}$ and its effect on the variation in the mobility of charge carriers. Interestingly, it is observed that the native defects like Oxygen vacancy has a prominent effect on the spectral features of Oxygen $\mathrm{K}$ edge and Cadmium $\mathrm{M}_{4,5}$ edge gives an evidence of $\mathrm{O} 2 p$-Cd $4 d$ hybridization. The growth of crystallites is understood by the expansion of lattice as results of replacement of $\mathrm{Cd}^{2+}$ ion by $\mathrm{Sn}^{2+}$ and liberation of electrons in the conduction band results an enhancement in band gap as well as in carrier concentration which also reduces the mobility. Thus, the present manuscript reports formation of charge neutrality level and its correlation with electronic structure and $p$ - $d$ hybridization is reported which has very interesting ramifications besides its crucial role for the development of optoelectronic devices.

\section{References}

1. Jefferson, P. H. et al. Bandgap and effective mass of epitaxial cadmium oxide. Appl. Phys. Lett. 92, 022101 (2008).

2. Cimino, A. \& Marezio, M. Lattice parameter and defect structure of cadmium oxide containing foreign atoms. J. Phys. Chem. Solids 17, 57-64 (1960).

3. Haul, R. \& Just, D. Disorder and oxygen transport in cadmium oxide. J. Appl. Phys. 33, 487-493 (1962).

4. Koffyberg, F. P. Electronic conduction and defect equilibria in CdO single crystals. J. Solid State Chem. 2, 176-181 (1970).

5. Tsukazaki, A. et al. Repeated temperature modulation epitaxy for p-type doping and light-emitting diode based on ZnO. Nat. Mater. 4, 42-46 (2005)

6. Coutts, T. J., Young, D. L., Li, X., Mulligan, W. P. \& Wu, X. Search for improved transparent conducting oxides: A fundamental investigation of CdO, $\mathrm{Cd}_{2} \mathrm{SnO}_{4}$, and $\mathrm{Zn}_{2} \mathrm{SnO}_{4}$. J. Vac. Sci. Technol. A 18, 2646-2660 (2000).

7. Finkenrath, H., Uhle, N. \& Waidelich, W. The influence of phonons and polarons on the infrared absorption of cadmium oxide. Solid State Commun. 7, 11-14 (1969).

8. Alford, T. L., Feldman, L. C. \& Mayer, J. W. Fundamentals of nanoscale film analysis. (Springer Science \& Business Media, 2007)

9. Cullity, B. D. Element of X-ray diffraction. Addition-Wesley. Reading, MA (1978).

10. Zheng, B. J., Lian, J. S., Zhao, L. \& Jiang, Q. Optical and electrical properties of Sn-doped CdO thin films obtained by pulse laser deposition. Vacuum 85, 861-865 (2011).

11. Schaeffer, C. D., Strausser, C. A., Thomsen, M. W. \& Yoder, C. H. Data for General, Inorganic, Organic, and Physical Chemistry (1989).

12. Velusamy, P., Babu, R. R., Ramamurthi, K., Viegas, J. \& Elangovan, E. Structural, microstructural, optical and electrical properties of spray deposited rare-earth metal (Sm) ions doped CdO thin films. J. Mater. Sci. Mater. Electron. 26, 4152-4164 (2015).

13. Singh, R. G., Singh, F., Kumar, V. \& Mehra, R. M. Growth kinetics of ZnO nanocrystallites: Structural, optical and photoluminescence properties tuned by thermal annealing. Curr. Appl. Phys. 11, 624-630 (2011).

14. Wang, Y., Liu, T., Wang, X. \& Song, H. Controlled synthesis of ytterbium ion and erbium ion codoped gadolinium oxyfluoride hollow nanosphere with upconversion luminescence property. J. Mater. Res. 28, 848-855 (2013).

15. Wright, M. \& Uddin, A. Organic-inorganic hybrid solar cells: A comparative review. Sol. energy Mater. Sol. cells 107, 87-111 (2012).

16. Xu, X., Lau, S., Chen, J., Chen, G. \& Tay, B. Polycrystalline ZnO thin films on Si (100) deposited by filtered cathodic vacuum arc. J. Cryst. Growth 223, 201-205 (2001).

17. Ziegler, E., Heinrich, A., Oppermann, H. \& Stöver, G. Electrical properties and non-stoichiometry in ZnO single crystals. Phys. status solidi 66, 635-648 (1981).

18. Tabet-Derraz, H., Benramdane, N., Nacer, D., Bouzidi, A. \& Medles, M. Investigations on Zn x Cd $1-x$ O thin films obtained by spray pyrolysis. Sol. energy Mater. Sol. cells 73, 249-259 (2002).

19. Moss, T. S. The interpretation of the properties of indium antimonide. Proc. Phys. Soc. Sect. B 67, 775 (1954).

20. Burstein, E. Anomalous optical absorption limit in InSb. Phys. Rev. 93, 632 (1954).

21. Ueda, N., Maeda, H., Hosono, H. \& Kawazoe, H. Band-gap widening of CdO thin films. J. Appl. Phys. 84, 6174 (1998).

22. Jaegermann, W. W. Mönch: Semiconductor Surfaces and Interfaces, Springer Series in Surface Science, Vol. 26, Springer-Verlag, Berlin Heidelberg New York 1995, ISBN 3-540-58625-3, DM 84,-, 442 Seiten. Berichte der Bunsengesellschaft für Phys. Chemie 100, 402 (1996).

23. Mönch, W. Electronic properties of semiconductor interfaces. 43 (Springer Science \& Business Media, 2013).

24. Piper, L. F. J. et al. Electronic structure of single-crystal rocksalt CdO studied by soft x-ray spectroscopies and ab initio calculations. Phys. Rev. B 77, 125204 (2008).

25. Jaffe, J. E., Pandey, R. \& Kunz, A. B. Electronic structure of the rocksalt-structure semiconductors ZnO and CdO. Phys. Rev. B 43, 14030 (1991).

26. King, P. D. C. et al. Unification of the electrical behavior of defects, impurities, and surface states in semiconductors: Virtual gap states in CdO. Phys. Rev. B 79, 035203 (2009).

27. Walukiewicz, W. Amphoteric native defects in semiconductors. Appl. Phys. Lett. 54, 2094-2096 (1989).

28. Tersoff, J. Band lineups at II-VI heterojunctions: failure of the common-anion rule. Phys. Rev. Lett. 56, 2755 (1986).

29. Walukiewicz, W. Mechanism of Fermi-level stabilization in semiconductors. Phys. Rev. B 37, 4760 (1988).

30. Sankey, O. F. Dangling bonds and Schottky barriers. J. Vac. Sci. Technol. B Microelectron. Nanom. Struct. 3, 1162 (1985).

31. King, P. D. C. et al. Surface electron accumulation and the charge neutrality level in In 2 O3. Phys. Rev. Lett. 101, 116808 (2008),

32. Rieder, K. H., Ishigame, M. \& Genzel, L. Infrared Absorption by Coupled Surface-Phonon-Surface-Plasmon Modes in Microcrystals of CdO. Phys. Rev. B 6, 3804 (1972).

33. Popović, Z. V., Stanišić, G., Stojanović, D. \& Kostić, R. Infrared and raman spectra of CdO. Phys. status solidi 165, K109-K112 (1991). 
34. Cuscó, R. et al. Raman scattering of cadmium oxide epilayers grown by metal-organic vapor phase epitaxy. J. Appl. Phys. 107, 063519 (2010).

35. Alim, K. A., Fonoberov, V. A., Shamsa, M. \& Balandin, A. A. Micro-Raman investigation of optical phonons in ZnO nanocrystals. J. Appl. Phys. 97, 124313 (2005).

36. Ashrafi, A. \& Ostrikov, K. K. Raman-active wurtzite $\mathrm{CdO}$ nanophase and phonon signatures in $\mathrm{CdO} / \mathrm{ZnO}$ heterostructures fabricated by nonequilibrium laser plasma ablation and stress control. Appl. Phys. Lett. 98, 133119 (2011).

37. Merlin, R., Pinczuk, A. \& Weber, W. H. In Raman scattering in materials science 1-29 (Springer, 2000).

38. Shi, W., Wang, C., Wang, H. \& Zhang, H. Hexagonal nanodisks of cadmium hydroxide and oxide with nanoporous structure. Cryst. Growth Des. 6, 915-918 (2006).

39. Demchenko, I. N. et al. Electronic structure of CdO studied by soft X-ray spectroscopy. J. Electron Spectros. Relat. Phenomena 184, 249-253 (2011).

40. McGuinness, C. et al. Influence of shallow core-level hybridization on the electronic structure of post-transition-metal oxides studied using soft X-ray emission and absorption. Phys. Rev. B 68, 165104 (2003).

41. Wei, S.-H. \& Zunger, A. Electronic structure of II-VI compounds and their alloys-role of cation d bands. J. Cryst. Growth 86, 1-7 (1988).

42. Wei, S.-H. \& Zunger, A. Role of metal d states in II-VI semiconductors. Phys. Rev. B 37, 8958 (1988)

43. Wei, S. \& Zunger, A. Electronic structure and stability of II-VI semiconductors and their alloys: The role of metal d bands. J. Vac. Sci. Technol. A 6, 2597-2611 (1988).

\section{Acknowledgements}

Authors are grateful to the Director (IUAC), Director (UGC-CSR Indore), Director (RRCAT, Indore) for their encouragement and moral support for extending experimental facilities including the synchrotron facility at RRCAT Indore. Authors are highly thankful to Dr. A. Roy (former Director, IUAC) for his critical reading for improving the quality of English throughout the manuscript. Authors are also thankful to Dr. (Mrs.) Indra Sulania, Dr. S. A. Khan and Mr. S. Ojha experimental supports in AFM, SEM and RBS measurements, respectively. Mr. Rakesh Sah (UGC-DAE CSR) is akcknowledged for his help during SXAS experiments at RRCAT, Indore. One of the authors (AD) acknowledges to Junior Research Fellowship (JRF) Grant Number-F.2-91/1998 (SA-1) from University Grant Commission, New Delhi. DST, Govt. Of India is also gratefully acknowledged for granting Science and Engineering Research Board (SERB) project (SB/EMEQ-122/2013).

\section{Author Contributions}

F.S. conceived the idea and planned the methodology of experiments. Experiments of synthesis, R.B.S., X.R.D., and UV-Vis, Hall measurements and micro-Raman spectroscopy were performed by A.D. and S.K.G. Soft x-ray absorption experiments and its analysis at BL-01 beamline of RRCAT, Indore were carried by A.D., S.K.G. and F.S. under the direction and expertise of D.K.S. Initial version of the manuscript was written by A.D. under the critical supervision of F.S. Later, all the authors have reviewed/corrected the manuscript and well aware about the submission of the manuscript.

\section{Additional Information}

Competing financial interests: The authors declare no competing financial interests.

How to cite this article: Das, A. et al. Correlations of charge neutrality level with electronic structure and p-d hybridization. Sci. Rep. 7, 40843; doi: 10.1038/srep40843 (2017).

Publisher's note: Springer Nature remains neutral with regard to jurisdictional claims in published maps and institutional affiliations.

(c) (i) This work is licensed under a Creative Commons Attribution 4.0 International License. The images or other third party material in this article are included in the article's Creative Commons license, unless indicated otherwise in the credit line; if the material is not included under the Creative Commons license, users will need to obtain permission from the license holder to reproduce the material. To view a copy of this license, visit http://creativecommons.org/licenses/by/4.0/

(C) The Author(s) 2017 